\title{
Automated Method for Optic Disc Detection and Elimination in Digital Fundus Images
}

\author{
Parashuram Bannigidad, Asmita Deshpande
}

\begin{abstract}
Localizing, segmenting and eliminating the optic disc region of a fundus image is a prerequisite task in the automatic investigation of a number of retinal diseases such as Diabetic retinopathy, Glaucoma, Macular Edema, etc. Accurate detection of optic disc is a challenging task due to a number of reasons. Optic disc in most fundus images does not exhibit clear disc boundaries and there are number of blood vessels crossing it. An important task in automated retinal image analysis system is the detection and elimination of optic disc because the lesion regions in diabetic retinopathy closely resemble the color and texture of an optic disc. Hence, eliminating the optic disc region can improve the performance of diabetic retinopathy detection. The proposed work presents a novel method for optic disc segmentation which is not restricted by the location of the optic disc on the retina. The proposed algorithm localizes the position of the optic disc that is independent of its location and dynamically finds its center. The proposed method is tested on images from DRISHTI-GS, DIARETDB1, DRIONS-DB and DRIVE databases based on morphological operation and finding the largest connected component. The precision values of segmentation for digital fundus images from DRISHTI-GS, DIARETDB1, DRIONS-DB, and DRIVE databases are 0.98, 0.99, 0.98 and 0.99 respectively using the proposed method. The algorithm has yielded consistent high values of precision and recall indicating its robustness and efficiency.
\end{abstract}

Keywords: fundus image, optic disc, morphological operations, diabetic retinopathy, segmentation, active contour, blood vessels.

\section{INTRODUCTION}

$\mathrm{M}$ illions of people around the world are affected by diabetes. It is a lifestyle disorder that affects the working of human organs such as kidneys, eyes, heart, and others. Diabetic patients suffer from a number of eye diseases that affect the retina such as diabetic retinopathy, glaucoma, macular degeneration, etc. A normal retinal image includes a number of anatomical structures such as retinal vasculature, optic disc, optic cup, and macula. The regular anatomical structures seen in a fundus image are presented in Fig.1. The optic disc is a yellowish circular region and a regular anatomical structure. Since there is an absence of rods and cones, the optic disc forms a blind spot on the human eye. An important task in automated retinal image analysis system is the detection and elimination of optic disc because the lesion regions in diabetic retinopathy closely resemble the color and texture of an optic disc. Its detection facilitates the segmentation of abnormal pathological features such as

Revised Manuscript Received on November 25, 2019.

* Correspondence Author Channamma University, Belagavi, Karnataka, India, parashurambannigidad@gmail.com

Asmita Deshpande*, Department of Computer Science, Belagavi, Karnataka, India, asd_bca@yahoo.com
Parashuram Bannigidad, Department of Computer Science, Rani

lesion, exudates and cotton wool spots which in turn improve the performance of algorithms that detect such retinal disorders.

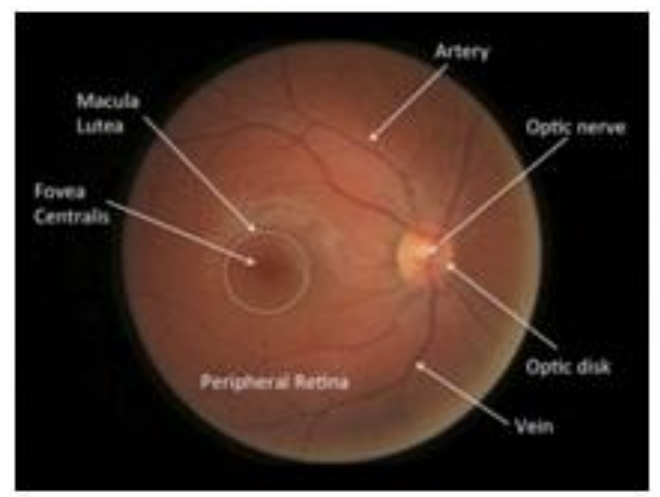

Fig. 1. A normal fundus image

Similarly, for the detection of another retinal disease called glaucoma the ratio of optic disc to cup diameter is measured. Many algorithms use location of the optic disc as a benchmark to measure the length in fundus images. Similarly for algorithms that detect blood vessels the position of the optic disc is important because most blood vessels originate from it. Digital fundus images help experts in the early detection of these diseases. Ophthalmologists can diagnose these diseases by examining the affected part of the retina. Usually, a non-mydriatic fundus camera is used to diagnose these disorders. The fundus camera helps understand the inside of the eye to locate the position of fovea, optic disc, macula, and the retina.

Several researchers have worked on retinal images for the location of the optic disc in a digital fundus image. Jinyang Sun et. al. [1] used image texture and active contour model for optic disc segmentation. Aniqa Azam et. al. [2] have applied a technique that is consisting of four important steps namely, preprocessing the fundus image, segmentation of vessels, extraction of bright region, and use of vessel density for optic disc detection. Wiseang et. al. [3] have implemented preprocessing techniques such as color normalization, contrast enhancement and noise removal followed by morphological operations and Otsu's algorithm, for optic disc detection. Mananis et. al. [4] have created a combined structure for analysis of fundus images and segmentation of retinal blood vessels as well as optic disc segmentation. They have implemented deep convolutional neural networks (CNNs), which have provided significant results in the area of computer vision particularly object detection and image classification. Akshaya Ramaswamy et. al. [5] have applied a unique method for detection of glaucoma based on representing the optic disc using a polar map which

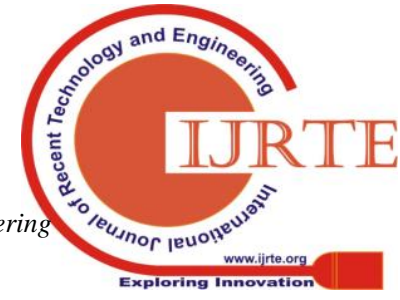


combines certain supervised and unsupervised cup segmentation techniques. Hemanth and Anitha [6] have applied a hybrid technique for segmentation that uses fuzzy and level set method. Juan $\mathrm{Xu}$ et. al. [7] have built a deformable-model based method that implements the active contour for knowledge-based clustering. It not only detects optic disc but also optic cup boundaries. Arturo Aquino et. al. [8] have detected the optic disc boundary by morphological and edge detection techniques subsequent by the Circular Hough. Mohammed Abdullah et. al. [9] have explored a powerful technique for optic disc detection and boundary segmentation using morphological operations, the circular Hough transform and the grow-cut algorithm. One of the important challenges in segmentation of optic disc is that though it is nearly circular in shape, its location is not always fixed. It may be located on the left or right border or even at the center. Hence, it is necessary to develop an algorithm that is not restricted by its location. In this paper, we propose an algorithm that is independent of the position of the optic disc and dynamically localizes its center.

\section{MATERIALS AND METHODS}

In this experiment, 101 digital fundus images from DRISHTI-GS[13] database were tested. It contains 50 training and 51 testing images. The database provides images wherein the optic disc has been marked manually by four experienced Ophthalmologists that serve as ground truth. The fundus images have been collected with the patient's consent from Aravind eye hospital, Madurai. Both male and female patients in the age group 40-80 years have been considered. These digital fundus images have been captured with 30 degrees FOV and $2896 \times 1944$ pixels resolution and are stored in PNG format. Images from another public database DIARETDB1[14] have also been tested in this experiment. It consists of 89 images that have been captured at 50 degree FOV with changing camera settings. This dataset has been benchmarked for detection of diabetic retinopathy. DRIONS-DB[18] is a public database created for benchmarking optic nerve head in retinal images. It comprises of 110 images. These images have a resolution of $600 \times 400$ and are represented using 8 bits/pixel. The images from DRIVE[17] database were obtained from patients in the age group 25-90. A 3CCD non-mydriatic camera was used to capture the images at 45 degree field-of-view having a resolution of $768 \times 584$ and represented with 8 bits per color plane.

\section{METHODOLOGY}

Localizing, segmenting and eliminating the optic disc region of a fundus image is a prerequisite task in automatic diagnosis of a number of retinal diseases such as Diabetic Retinopathy, Glaucoma, Macular Edema, etc. A study of fundus images gives deep understanding of the various anatomical structures. Fundus images exhibit optic disc with varied sizes, but mostly it has been observed that its diameter is between 80 and 100 pixels. Accurate detection of optic disc is a challenging task due to a number of reasons. Optic disc in most fundus images does not exhibit clear disc boundaries and there a number of blood vessels crossing it. In this experiment two segmentation techniques, namely, active contour and k-Means clustering were used along with the proposed method for detection of optic disc.

\section{i. Active Contour Method}

Contours are nothing but curves that join continuous points having the same color or intensity along a boundary. The contours mechanisms are used in shape analysis and pattern recognition. Active contours enable image processing through which deformable models or structures can be obtained for segmentation [21].

\section{ii. k-Means Clustering}

$\mathrm{k}-$ Means is an unsupervised machine learning algorithm. Such algorithms draw their inferences from datasets using only input vectors without having any known outcomes. It finds $\mathrm{k}$ number of centroids and then assigns every data point to the nearest cluster [16].

\section{iii. Proposed Method}

The methodology followed in this paper comprises of two important steps. Initially, the approximate position of the optic disc is localized and then its center is found using repeated thresholding and connected component method [16].

\section{A. Preprocessing}

Digital fundus images are characterized by irregular illumination. Preprocessing can not only reduce the unequal illumination but also focus on smaller details in the retinal image such as blood vessels, lesions, and exudates. It is commonly observed that there is more contrast at the center of a fundus image and less towards the outer periphery of the image. The preprocessing stage prominently resizes the input image to standard size of 512 X 512 and further transforms it into grayscale. In order to enhance the contrast between foreground and background, image intensity level is mapped to higher intensities. Further, CLAHE is applied to adjust the contrast and distinguish between disc and non-disc region Fig. 2(a) depicts the grayscale image and the contrast adjusted image is depicted in Fig. 2(b). Edges in the fundus image are highlighted by applying canny edge detection. This is followed by detection and masking of the peripheral circular border of the fundus image and thresholding to binarize it.

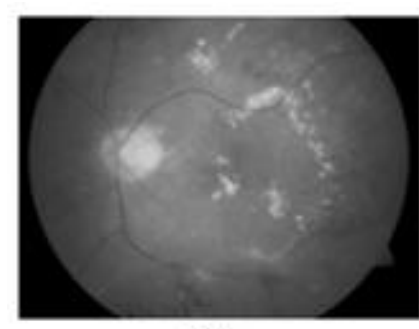

(a)

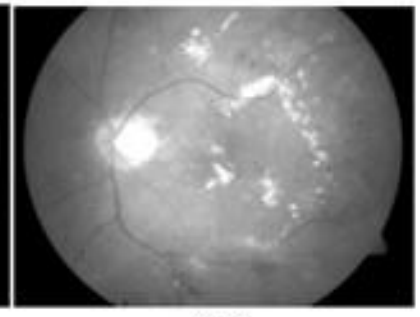

(b)
Fig. 2. (a) Grayscale image (b) Contrast adjusted image

\section{B. Blood Vessel Removal}

The retina comprises of a network of blood vessels which can deter accurate detection of the optic disc. Hence, it is necessary to detect and eliminate the complete blood vessel network. A disc shaped structuring element is chosen and morphological opening and closing operations [16] are applied to detect and eliminate the vessel path as follows: 


$$
\begin{gathered}
A(x, y)=(I \oplus S)(x, y)=\max \left\{I\left(x-x^{\prime}, y^{-y^{\prime}}\right)+S\left(x^{\prime}, y^{\prime}\right)\right\} \\
B(x, y)=(I \ominus S)(x, y)=\min \left\{I\left(x+x^{\prime}, y^{\prime} y^{\prime}\right)-S\left(x^{\prime}, y^{\prime}\right)\right\} \\
I_{n}=A(x, y)-B(x, y)
\end{gathered}
$$

where, $\mathrm{I}(\mathrm{x}, \mathrm{y})$ is the image obtained after external border masking during preprocessing and $\mathrm{S}\left(\mathrm{x}^{\prime}, \mathrm{y}^{\prime}\right)$ is the structuring element. These alternating opening and closing operations are used to remove unwanted components from the fundus image. Further, a ball shaped structuring element is used and morphological closing operation is executed to eliminate the vasculature completely. The image obtained after the removal of blood vessels is shown in Fig. 3.

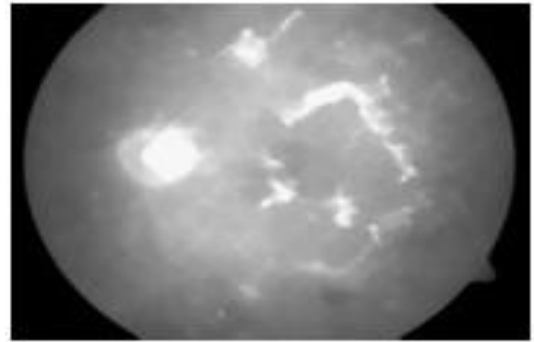

Fig. 3. Image after blood vessel removal

\section{Optic Disc Segmentation}

The proposed work is based on local threshold segmentation [21] because just like variation in intensity of the image, variation in the image foreground and background texture is also observed in the fundus image. Column-wise neighborhood operation is performed on fundus image In that is obtained in the previous step after elimination of blood vessels. Now, a fixed threshold is applied to filter the region with higher intensities.

$$
\begin{aligned}
& \mathrm{I}(\mathrm{x}, \mathrm{y})=1 \text { if } \mathrm{p}(\mathrm{x}, \mathrm{y})>T \\
& \mathrm{I}(\mathrm{x}, \mathrm{y})=0 \text { if } \mathrm{p}(\mathrm{x} . \mathrm{y})<T
\end{aligned}
$$

where, $\mathrm{T}=0.45$.

As mentioned earlier the optic disc is a large yellowish circular region. Hence, location on the disc is based on the discovery of the largest connected component in the grayscale image. This largest connected component represents the optic disc. The largest value of the columns of image and its coordinates is retrieved. The mask is computed with mesh grid vectors $\mathrm{x}$ and $\mathrm{y}$ as well as the medians of row $(\mathrm{mr})$ and column $(\mathrm{mc})$ based on the equation (6) of a circle:

$$
I m=\sqrt{(x-m c) 2-(y-m r) 2}
$$

The mask is subtracted from the binarized image to obtain the segmented image.

$$
I x y=I(x, y)-I m
$$

Fig. 4(a) shows the original image and its corresponding segmented image is shown in Fig. 4(b).

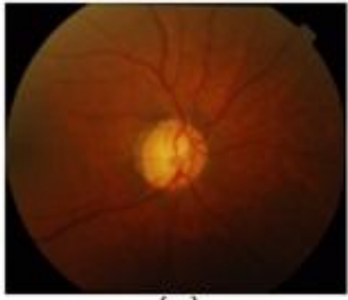

(a)

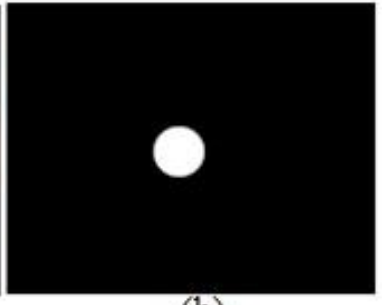

(b)
Fig. 4(a) Original image (b) Segmented image
The algorithm for the proposed method is described below:

\section{Algorithm}

Input : Color fundus image.

Output : Optic disc located and segmented.

Step 1. Resize the image to $512 \times 512$.

Step 2. Convert to grayscale.

Step 3. Apply CLAHE

Step 4. Create a mask and eliminate the outer circular border

Step 5. Apply morphological opening and closing operations to eliminate the blood vessels.

Step 6. Apply local thresholding to the image obtained in step 5 and filter the regions with higher intensities.

Step 7. Apply column wise neighbourhood operations to the image obtained in step 6 .

Step 8. Create a circular mask with mesh grid vectors $\mathrm{x}$ and $\mathrm{y}$ and the medians of the rows and columns.

Step 9. Subtract the mask from the image obtained in step 8 to obtain the final segmented image.

\section{EXPERIMENTAL RESULTS AND DISCUSSIONS}

This experiment was carried out on the digital fundus images obtained from publicly available databases, namely, DRISHTI-GS, DIARETDB1, DRIONS-DB, and DRIVE. In all, 340 fundus images having varying resolution were tested, out of which 101 images form DRISHTI-GS, 89 images from DIARETDB1, 110 from DRIONS-DB and 40 images from DRIVE were used. The proposed algorithm is coded in MATLAB R2015b. To compare the proposed segmentation technique, two other standard segmentation techniques, that is, Active contour segmentation and k-Means clustering were also considered. Fig. 5(a) shows sample original digital fundus images form DRISHTI-GS database. The segmented images are shown in Fig. 5(b); b(i) active contour method, b(ii) k-Means clustering and b(iii) Proposed method. The Fig. 6(a) shows sample original digital fundus images form DIARETDB1 database. The images with segmented region of interest are shown in Fig. 6(b). b(i) active contour method, $\mathrm{b}$ (ii) k-Means clustering and b(iii) Proposed method. Similarly, the Fig. 7(a) shows digital fundus images form DRIONS-DB database. The segmented images are shown in Fig. 7(b); b(i) active contour method, b(ii) k-Means clustering and b(iii) Proposed method. Fig. 8(a) shows digital fundus images form DRIVE database. The segmented images are shown in Fig. 8(b); b(i) active contour method, b(ii) k-Means Clustering and b(iii) Proposed method .

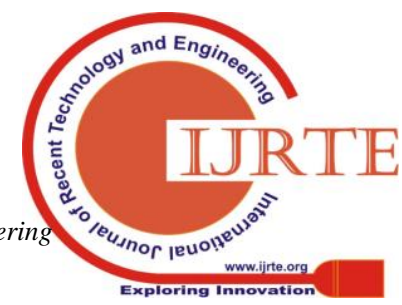


Automated Method for Optic Disc Detection and Elimination in Digital Fundus Images

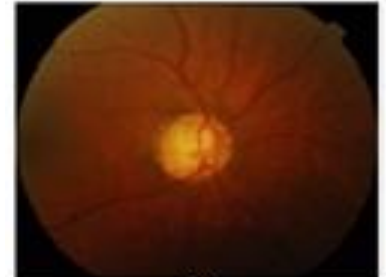

a(1)

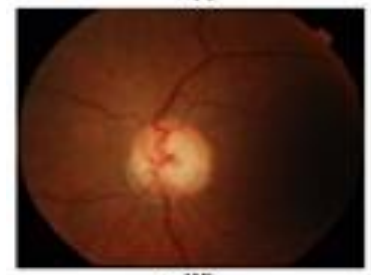

a(ii)

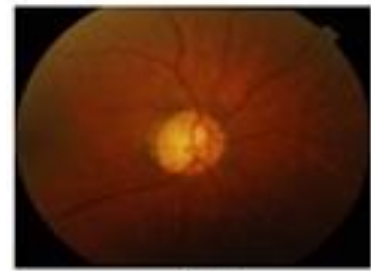

$\mathrm{a}$ (iii)

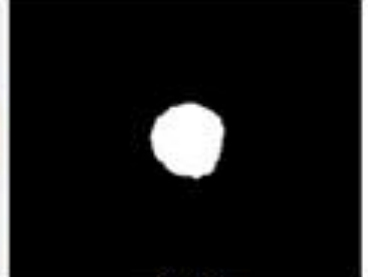

b(i)

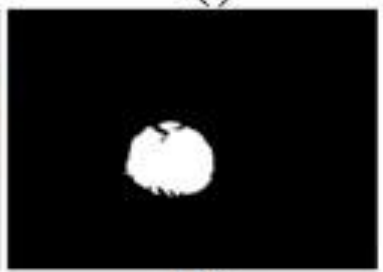

b(ii)

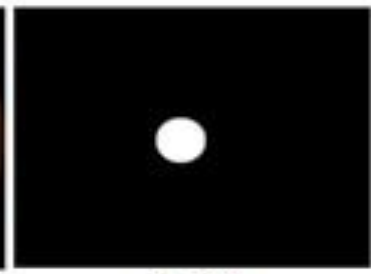

$\mathrm{b}$ (iii)

Fig 5. (a) Digital fundus images form DRIHSTI- GS; (b) Segmented images of (a); b(i) Active contour method;b(ii) k-Means clustering; b(iii) Proposed method

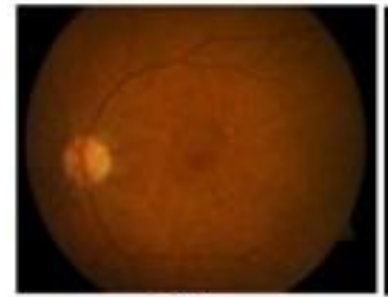

$\mathrm{a}(\mathrm{i})$

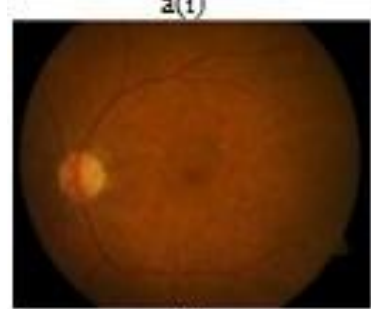

a(ii)

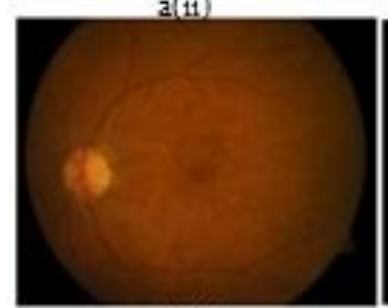

a(iii)

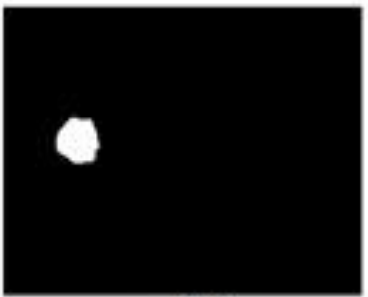

$b(1)$

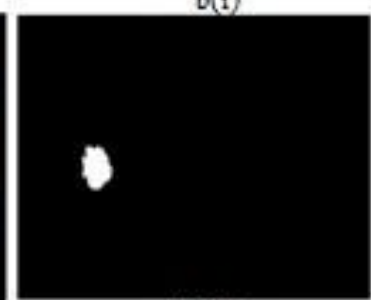

b(ii)

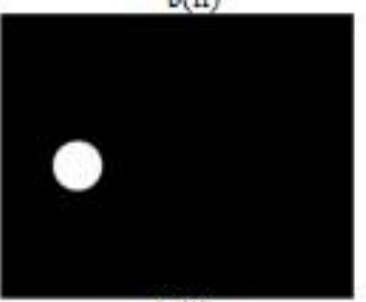

b(iii)

Fig 6. (a) Digital fundus images from DIARETDB1; (b)

Segmented images of (a);b(i) Active contour method; b(ii) k-Means clustering; b(iii) Proposed method

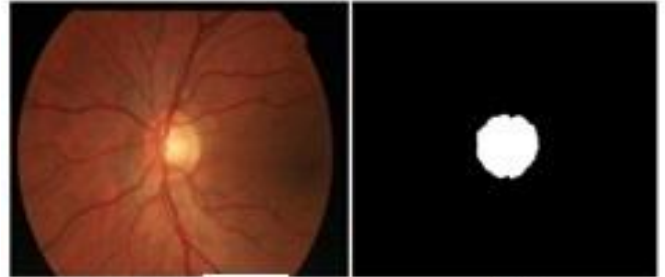

$a(i)$

b(i)

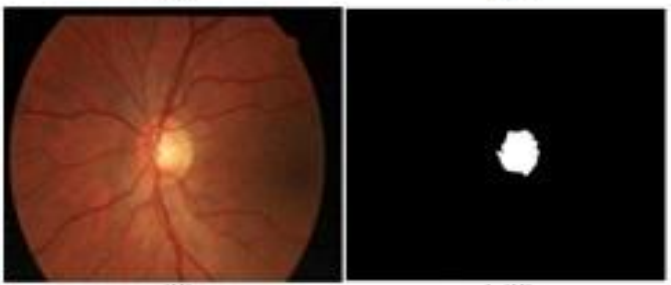

a(ii)

b(ii)

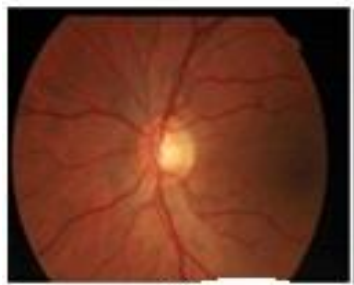

a(iii)

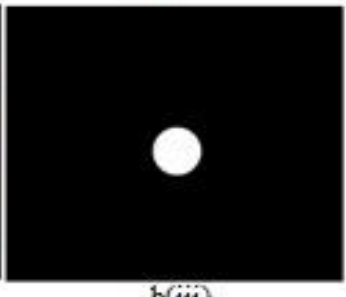

ages form

DRIONS-DB; (b) Segmented images of (a);b(i)

Active contour method; b(ii) k-Means clustering; b(iii) Proposed method;
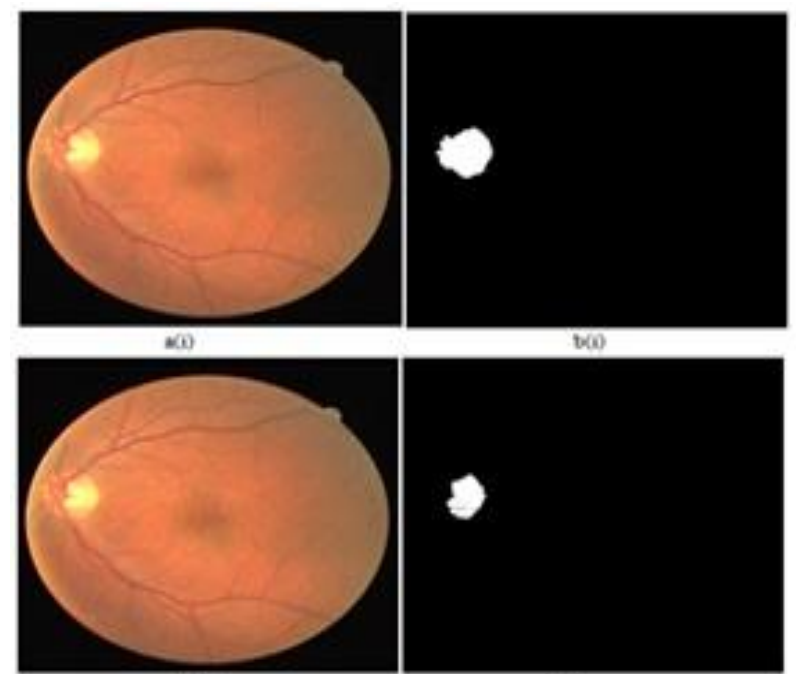

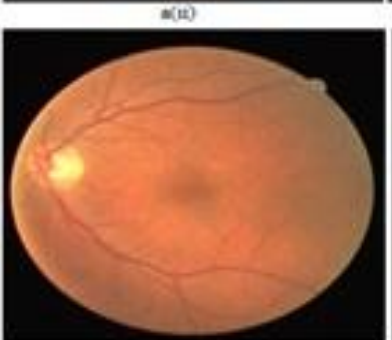

sing

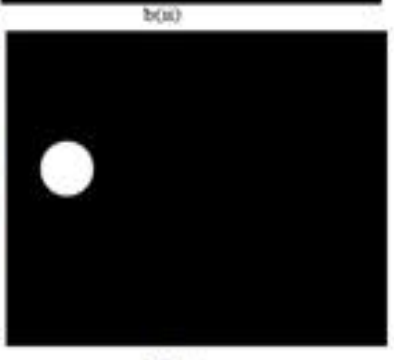

tones
Fig 8. (a) Digital fundus images form DRIVE; (b) Segmented images of (a); b(i) Active contour method; b(ii) k-Means clustering; b(iii) Proposed method;

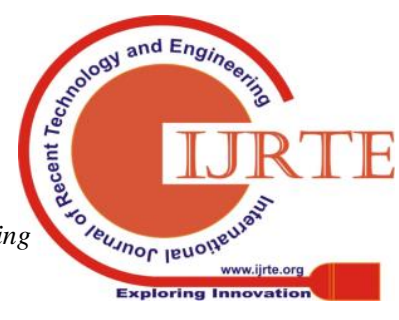


Table- I: Results of performance evaluation measures

\begin{tabular}{|c|c|c|c|c|c|c|c|c|c|c|c|c|}
\hline & \multicolumn{3}{|c|}{ DRISHTI-GS } & \multicolumn{3}{|c|}{ DIARETDB1 } & \multicolumn{3}{|c|}{ DRIONS-DB } & \multicolumn{3}{|c|}{ DRIVE } \\
\hline $\begin{array}{l}\text { Performance } \\
\text { Evaluation } \\
\text { Measures }\end{array}$ & $\begin{array}{l}\text { Active } \\
\text { Contour }\end{array}$ & k-Means & $\begin{array}{c}\text { Proposed } \\
\text { Method }\end{array}$ & $\begin{array}{l}\text { Active } \\
\text { Contour }\end{array}$ & k-Means & $\begin{array}{c}\text { Proposed } \\
\text { Method }\end{array}$ & $\begin{array}{l}\text { Active } \\
\text { Contour }\end{array}$ & k-Means & $\begin{array}{c}\text { Proposed } \\
\text { Method }\end{array}$ & $\begin{array}{l}\text { Active } \\
\text { Contour }\end{array}$ & k-Means & $\begin{array}{l}\text { Proposed } \\
\text { Method }\end{array}$ \\
\hline Precision & 0.98 & 0.98 & 0.98 & 0.98 & 0.99 & 0.99 & 0.98 & 0.97 & 0.98 & 0.98 & 0.99 & 0.99 \\
\hline Recall & 0.98 & 0.98 & 0.99 & 0.98 & 0.94 & 0.98 & 0.98 & 0.97 & 0.99 & 0.98 & 0.98 & 0.99 \\
\hline F-measure & 98.5 & 98.58 & 99.18 & 98.82 & 96.78 & 99.2 & 98.24 & 97.55 & 99.01 & 98.67 & 98.7 & 99.3 \\
\hline Accuracy & 0.97 & 0.97 & 0.98 & 0.97 & 0.94 & 0.98 & 0.96 & 0.95 & 0.98 & 0.97 & 0.97 & 0.98 \\
\hline PSNR & 16.05 & 17.87 & 18.22 & 16.81 & 15.61 & 18.88 & 15.29 & 13.25 & 17.31 & 16.87 & 18.09 & 19.15 \\
\hline MSE & 0.02 & 0.026 & 0.015 & 0.02 & 0.05 & 0.01 & 0.03 & 0.04 & 0.01 & 0.02 & 0.02 & 0.01 \\
\hline
\end{tabular}

The performance evaluation approaches namely, Precision, Recall, F-Measure, Accuracy, PSNR, and MSE, are used to evaluate the segmented images by comparing them with the ground truth images, and these are described below $[15,17,19,20,22,23]$ :

a) Precision: Precision represents the fraction of correct instances among the retrieved instances in pattern recognition and classification.

$$
\text { Precision }=\mathrm{TP} / \mathrm{TP}+\mathrm{FP}
$$

b) Recall: It is the ratio of True Positives (TP) to the sum of True Positives and False Negatives (FN).

$$
\text { Recall }=\mathrm{TP} / \mathrm{TP}+\mathrm{FN}
$$

Hence, high precision means that an algorithm is efficient in returning relevant results, while high recall value indicates that an algorithm was successful in returning most of the relevant results.

c) F-Measure: The harmonic mean of precision and Recall is known as F-Measure.

$$
\mathrm{F}=2 \cdot \frac{\text { Precision. Recall }}{\text { Precision }+ \text { Recall }}
$$

d) Accuracy: It represents a measure of the proportion of the sum of True Positives and True Negatives to the total population.

$$
\mathrm{Ac}=\frac{\mathrm{TN}+\mathrm{TP}}{\mathrm{TP}+\mathrm{FP}+\mathrm{TN}+\mathrm{FN}}
$$

where, TP is True Positives, FP is False Positives, TN is True Negatives and FN is False Negatives.

e) Peak Signal to Noise Ratio (PSNR): It is the proportion between the maximum power of a signal to the power of noise that corrupts the representation of the original signal

$$
P S N R=10 \cdot \log \left(\frac{\mathrm{MAX} 1^{2}}{\mathrm{MSE}}\right)
$$

f) Mean Square Error (MSE): This metric is used to measure the quality of the image.

$$
M S E=\frac{1}{M S E m n} \sum_{i=0}^{m-1} \sum_{j=0}^{n-1}[I(i, j)-K(i, j)]
$$

where, $I(i, j)$ and $K(i, j)$ represent the segmented and the ground truth images respectively.

The results obtained after applying the various performance evaluation measures on the segmented images by comparing them with ground truth images are depicted in
Table - I. The results described in above Table - I show that though all the three methods have produced good results, the proposed method has produced best results of segmenting the optic disc in retinal fundus images. The performance evaluation measures namely, Precision, Recall, F-measure, Accuracy, PSNR and MSE for the proposed method were obtained as $0.98,0.99,99.18,0.98,18.22,0.015$ for DRISHTI-GS， 0.99，0.98，99.20，0.98， 18.88，0.01 for DIARETDB1， 0.98， 0.99，99.01， $0.9817 .31,0.01$ for DRIONS-DB and $0.99,0.99,99.30,0.98,19.15,0.01$ for DRIVE databases respectively.

\section{CONCLUSION}

Optic Disc segmentation and elimination are integral to the detection of a number of retinal diseases such as Diabetic retinopathy, Glaucoma and Diabetic maculopathy. This paper presents a novel method for optic disc segmentation which is not restricted by the location of the optic disc on the retina. Initially, the approximate position of the optic disc is localized and then its centre is found using repeated thresholding, morphological operations and connected component method. Thus, the proposed algorithm is independent of the position of the optic disc and dynamically localizes its centre. The proposed method was tested on fundus images with different resolution from various databases. The performance evaluation measures namely, Precision, Recall, F-measure, Accuracy, PSNR and MSE for the proposed method were obtained as $0.98,0.99,99.18$, 0.98, 18.22, 0.015 for DRISHTI-GS, 0.99, 0.98, 99.20, 0.98, 18.88, 0.01 for DIARETDB1, 0.98, 0.99, 99.01, 0.98 17.31, 0.01 for DRIONS-DB and 0.99, 0.99, 99.30, 0.98, 19.15, 0.01 for DRIVE databases respectively. The algorithm has yielded consistent high values of Precision and Recall indicating its robustness and efficiency.

\section{ACKNOWLEDGEMENT}

The authors are indebted to the developers of DRISHTI-GS, DIARETDB1, DRIONS-DB and DRIVE databases for making digital fundus images freely and publicly available to the research community. 


\section{REFERENCES}

1. G. Jinyang Sun, Fangjun Luan and Hanhui Wu, "Optic Disc Segmentation by Balloon Snake with Texture from Color Fundus Image", International Journal of Biomedical Imaging, 2015, pp. 1-14.

2. Aniqa Azam, M. Usman Akram, Usman Qamar, "Optic Disc Segmentation from Colored Retinal Images using Vessel Density", IEEE International Conference on Frontiers of Information Technology, 2014, pp. 317-318.

3. Kittipol Wisaeng, Nualsawat Hiransakolwong, Ekkarat Pothiruk, "Automatic Detection of Optic Disc in Digital Retinal Images", International Journal of Computer Applications, Volume 90, No 5, 2014, pp. $15-20$.

4. Kevis-Kokitsi Maninis, Jordi Pont-Tuset, Pablo Arbel'aez, and Luc Van Gool, "Deep Retinal Image Understanding”, Medical Image Computing and Computer-Assisted Intervention (MICCAI), 2016.

5. Ramaswamy, Akshaya; Ram, Keerthi; Joshi, Niranjan; and Sivaprakasam, Mohanasankar, "A Polar Map Based Approach Using Retinal Fundus Images for Glaucoma Detection" In: Chen X, Garvin MK, Liu JJ, Trusso E, Xu Y editors. Proceedings of the Ophthalmic Medical Image Analysis Second International Workshop, OMIA 2015, Held in Conjunction with MICCAI 2015, pp.145-152.

6. J. Hemanth and J. Anitha, "Hybrid clustering method for optic disc segmentation and feature extraction in retinal images", World Congress on Information and Communication Technologies, Trivandrum, 2012, pp. 320-325.

7. Juan Xua,, Opas Chutatapeb, Eric Sungc, Ce Zhengd, Paul ChewTec Kuand, "Optic disk feature extraction via modified deformable model technique for glaucoma analysis", Pattern Recognition, Vol. 40, Issue 7, 2007, pp. 2063-2076.

8. Arturo Aquino*, Manuel Emilio Gegúndez-Arias, and Diego Marí, "Detecting the Optic Disc Boundary in Digital Fundus Images Using Morphological, Edge Detection, and Feature Extraction Techniques", IEEE transactions on medical imaging, Volume. 29, no. 11, 2010, pp. 1860-1869.

9. Muhammad Abdullah, Muhammad Moazam Fraz, and Sarah A.Barman, "Localization and segmentation of optic disc in retinal images using circular Hough transform and grow-cut algorithm", PeerJ4:e2003;DOI10.7717/peerj.2003

10. Parashuram Bannigidad and Asmita Deshpande, " A Hybrid Approach for Digital Fundus Images using Image Enhancement Techniques", International Journal of Computer Engineering and Applications, Vol. XII, Issue I, 2017, pp.122-131.

11. Parashuram Bannigidad and Asmita Deshpande, "A Multistage Approach for exudates detection in fundus images using texture features with k-NN classifier", International Journal of Advanced Research in Computer Science, Vol. 9, No. 1, 2018, pp.1-5.

12. Parashuram Bannigidad and Asmita Deshpande, "Exudates Detection in Digital Fundus Images using GLCM features with SVM classifier", International Journal of Modern Electronic and Communication Engineering, Vol. 6, Issue. 6, 2018, pp.184-189.

13. J. Sivaswamy, S. R. Krishnadas, G. Datt Joshi, M. Jain and A. U. Syed Tabish, "Drishti-GS: Retinal image dataset for optic nerve head(ONH) segmentation", 2014, IEEE 11th International Symposium on Biomedical Imaging (ISBI), Beijing, 2014, pp. 53-56.

14. http://www..it.lut.fi/project/imageret/diaretdb1/

15. https://en.wikipedia.org/wiki/Sensitivity_and_specificity

16. Raefel C Gonzalez, Richard E. Woods, "Digital Image Processing”, 3rd Edition, 2014.

17. J.J. Staal, M.D. Abramoff, M. Niemeijer, M.A. Viergever, B. van Ginneken, "Ridge based vessel segmentation in color images of the retina", IEEE Transactions on Medical Imaging, 2004, vol. 23, pp. 501-509.

18. E.J. Carmona, M. Rincón, J. García-Feijoo and J. M. Martínez-de-la-Casa, "Identification of the optic nerve head with genetic algorithms", Artificial Intelligence in Medicine, 2008,Vol. 43(3), pp. 243-259

19. https://en.wikipedia.org/wiki/Precision_and_recall

20. https://en.wikipedia.org/wiki/Peak_signal-to-noise_ratio

21. Tony Chan, Luminita Vese, "Active Contour without Edges", IEEE Transactions on Image Processing, Vol 10, No. 2, 2001, pp. 266-277

22. Parashuram Bannigidad and Chandrashekar Gudada, "Restoration of Degraded Historical Kannada Handwritten Document Images using Image Enhancement Techniques", International Conference on Soft Computing and Pattern Recognition (SoCPaR 2016), 2016. pp. 498-508

23. Parashuram Bannigidad and Chandrashekar Gudada," Restoration of Degraded Kannada Handwritten Paper Inscriptions (Hastaprati) using Image Enhancement Techniques", IEEE International Conference on Computer Communication and Informatics (ICCCI -2017), 2017. pp.1-6.

\section{AUTHORS PROFILE}

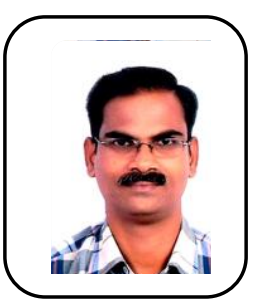
by VGST Govt. of Karnataka.

Dr. Parashuram Bannigidad is an Assistant Professor in the Department of Computer Science, Rani Channamma University, Belagavi, Karnataka, India. He is specialized in image processing and Pattern Recognition, Biomedical, Document image analysis and Nanotechnology. He has published more than 60 research papers in International Journals and Conference proceedings. $\mathrm{He}$ has completed four research projects funded by Govt. of Karnataka, UGC, New Delhi. He also received Young Scientist Award

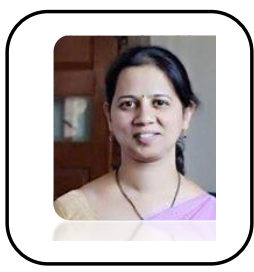

Asmita S. Deshpande received her B.Sc in Computer Science from Karnatak University, Dharwad and MCA from IGNOU, New Delhi in 1998 and 2009, respectively. She is working as a Lecturer in Gogte BCA College, Belagavi, since 2004. Presently she is pursuing her Ph.D. in Computer Science from Rani Channamma University, Belagavi, Karnataka, India. She is specialized in medical image processing and pattern recognition. She has published more than 5 research papers in International Journals. 